\title{
Fast timing detectors with applications in cosmic ray physics and medical science
}

\author{
Christophe Royon ${ }^{1}$ \\ ${ }^{1}$ Department of Physics and Astronomy, The University of Kansas, Lawrence, USA \\ Christophe.royon@cern.ch
}

\begin{abstract}
We describe two applications concerning fast timing detectors and fast signal sampling in cosmic ray measurements in collaboration with NASA and in dose measurements for cancer treatment.
\end{abstract}

Keywords - cosmic ray measurement, radiation measurement, space physics, dose measurement in medicine.

\section{INTRODUCTION: SIGNAL AMPLIFICATION AND MEASUREMENT}

$\mathrm{T}^{\mathrm{H}}$ HE idea is to discuss some applications to measure radiations using Fast Silicon detectors that are used in a standard way nowadays in high energy physics at CERN $[1,2,3]$. We will first describe the principle and methods of the technics that we use and describe in detail two applications related to measurements of cosmic ray in space in collaboration with NASA and of doses received by patients in cancer treatment especially in flash proton therapy.

The principle behind the measurements is shown in Fig. 1. We first use a Silicon detector in order to detect particles that go through the detector. In particular, we use fast detectors so that the signal emitted by the particle has a short time length, typically a few nanoseconds. This has the main advantage of

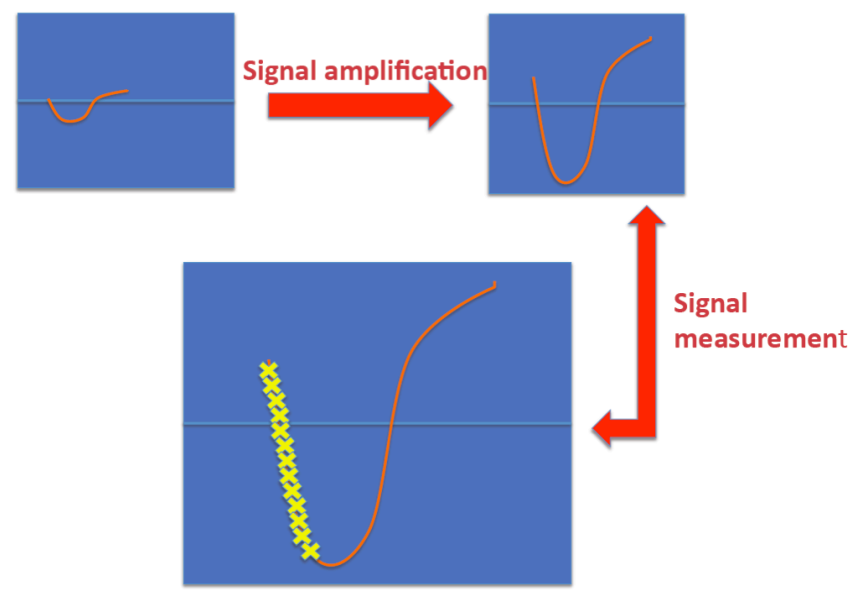

Fig. 1. Principle of signal analysis. A particle entering a fast Silicon detector induces a signal that is first amplified and then analyzed by performing a fast sampling of the signal (yellow crosses) that allow to measure the arrival time of the signal, its amplitude, shape and duration. reducing the probability of overlaps between the signal produced by many particles.

The signal originating from the Silicon detector (or the diamond detector) is first amplified with very limited shaping (so without modifying the good timing properties of the signal such as its duration). This is performed using front end electronics designed at the University of Kansas using standard components [4]. The following step consists in performing a fast sampling and digitization of the signal as shown in Fig. 1. This presents two advantages. First, it allows obtaining very precisely a time stamp when the signal (or in other words the particle) arrives in the detector (this is used to measure time-of-flight of particles in high energy physics with a precision of 15-20 picoseconds). Second, it allows reconstructing the full amplitude and shape of the signal by performing an interpolation between the sampled points (there can be 64 points in a few nanoseconds) if the signal is short in time.

We installed a full test stand at the University of Kansas allowing to test the performance of the Si detectors and their electronics and preliminary measurements showed resolutions between 15 and 20 picoseconds for ultra fast Silicon detectors as an example.

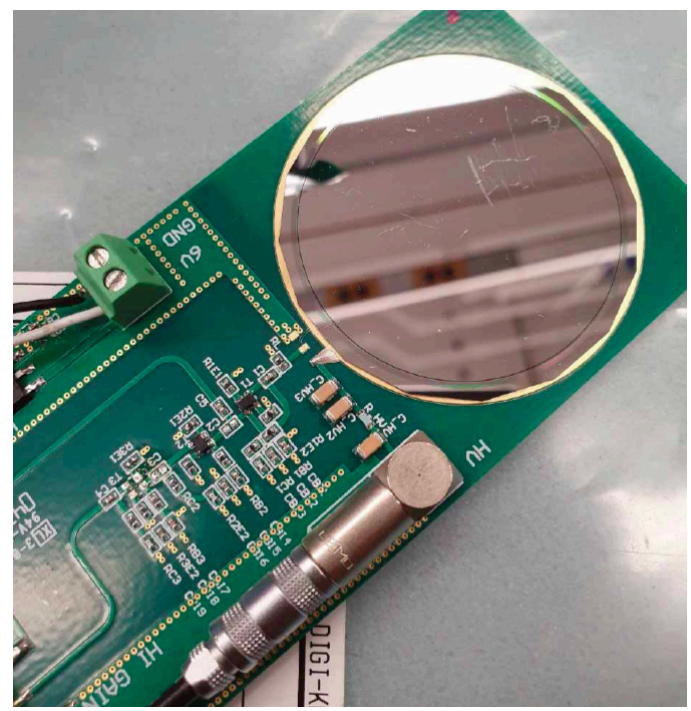

Fig. 2.View of the Silicon detector to be sent into space in collaboration with NASA (AGILE project) together with the readout electronics built in Kansas. 


\section{MEASURING COSMIC RAY IN SPACE: THE AGILE PROJECT}

The goal of the AGILE project in collaboration with NASA [5] is to measure cosmic ray particles in space in the MeV$\mathrm{GeV}$ range. The goal is to identify the type of particle (electron, proton, $\mathrm{He}, \mathrm{Pb}, \mathrm{Au} . .$. ) and at the same time measure their energy in space using a compact and affordable detector that can be part of a cube satellite. The basic idea is to use different layers of fast Silicon detector and to measure and reconstruct the signal where the particle stops in a given layer, the so called Bragg peak, using the fast sampling technique. A view of one layer of the detector to be sent into space together with the readout electronics is shown in Fig. 2.

The results discussed in this section come from a direct simulation of the signal using GEANT (particle simulation and interaction with the detector), WEIGHTFIELD (simulation of the silicon detector) and LTSPICE (simulation of electronics) as shown in Fig. 3. The output of this simulation is to obtain a database of signal characteristics that lead to particle identification and energy measurement.

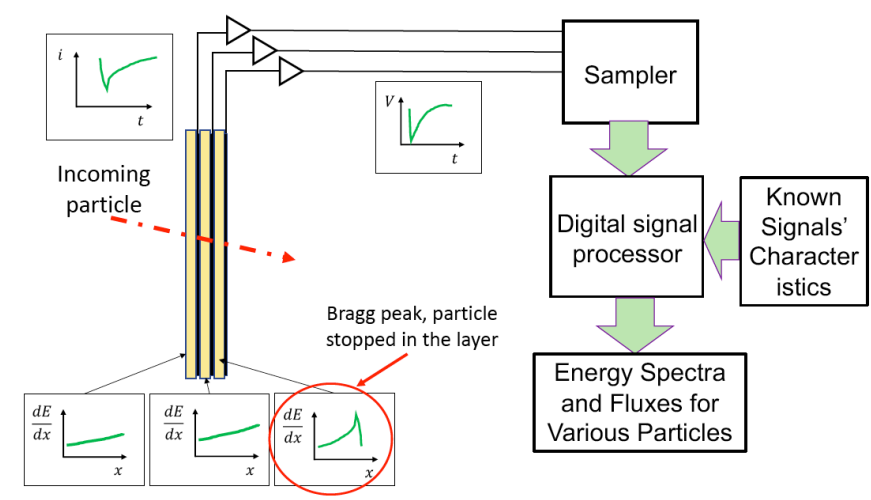

Fig. 3. Schematic of the simulation of the events measured by AGILE. We first use te signal collected by three layers of the fast Silicon detector. We use a fast sampling method to sample that signal, which is then digitized. It leads to a database of energy spectra and fluxes for different particles that allow to define the best characteristics for particle identification and energy measurement.

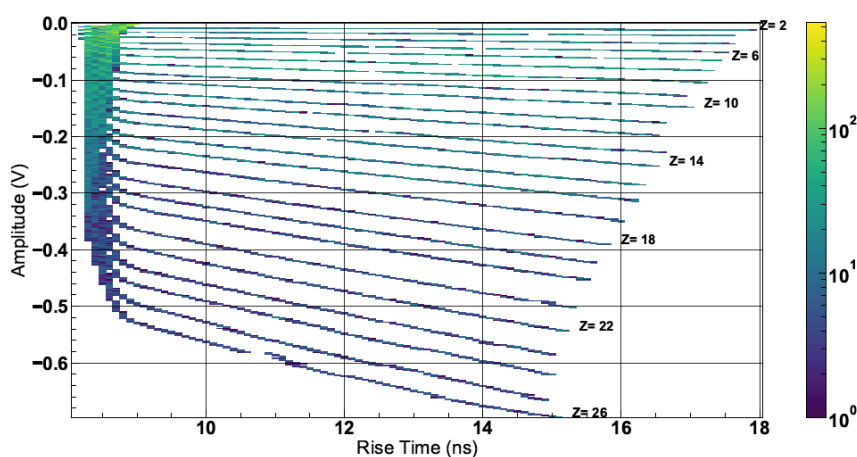

Fig. 4. Amplitude versus rise time for different particles (the value of $\mathrm{Z}$ is indicated on the figure). Above a rise time of about $6 \mathrm{~ns}$, the curves are well separated which shows that it is possible to distinguish between different particle species and to measure their energy.

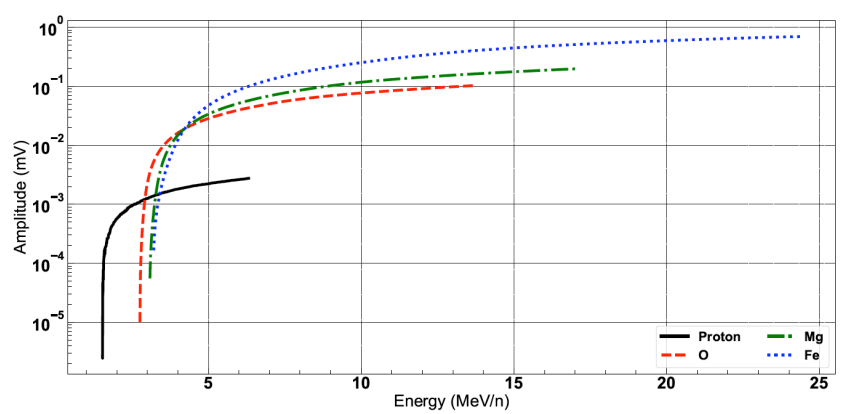

Fig. 5. Amplitude versus energy for different kinds of particles (proton, oxygen, magnesium and iron). If we assume that the kind of particle is known from the rise time measurement (see Fig. 2), the amplitude allows measuring the energy of the particle on a wide range above $5 \mathrm{MeV}$.

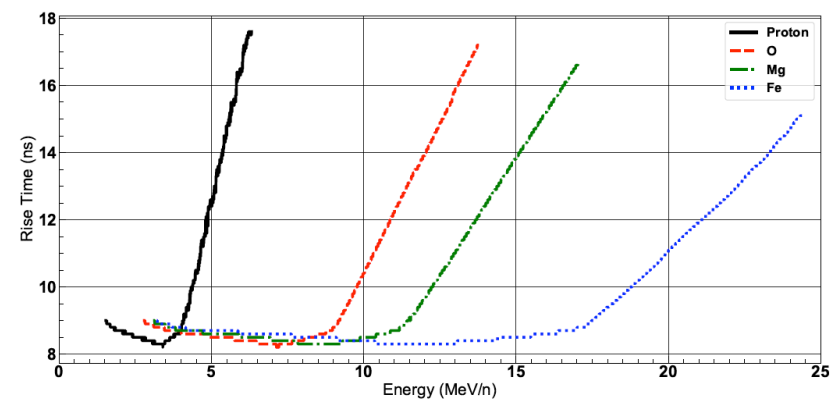

Fig. 6. Rise time versus energy for different kinds of particles (proton, oxygen, magnesium and iron). We see that the type of particle can be easily identified over a wide domain in energy by measuring the rise time of the signal.

In order to identify the type of particle and to measure their energy, we use two estimators. The first one is the rise time and the second one the amplitude of the signal, both measured in the layer where the particle stops (from the trigger point of view, we will require no signal in the next layer).

The amplitude as a function of rise time in the stopping layer, as put in the reference database, is shown in Fig. 4 for different particles with $Z$ between 2 and 28 . We see that all curves (corresponding to different values of $Z$ ) do not overlap for a rise time above $5 \mathrm{~ns}$. It is thus clear that particle identification or in other words, the measurement of the $\mathrm{Z}$ value, can be performed using the rise time and amplitude of the signal. Once the type of the particle is known, it is possible to measure its energy using the amplitude of the signal as shown in Fig. 5 for proton, oxygen, magnesium and iron. Energy measurements can clearly be performed for all kinds of heavy ions in space above about $5 \mathrm{MeV}$ with good resolution. The rise time versus energy of the particle is shown on Fig. 6 for the same particles. We clearly see the reason why the rise time has to be above $5 \mathrm{~ns}$ due to the shape of the curves.

The two measurements of the signal amplitude and rise time can be performed online in the cube satellite using a processor 
or offline on earth after data have been transmitted. The launch of the satellite is foreseen for Spring 2022 with three layers of Silicon detectors only as a prototype. The goal is to have a detector fully approved by NASA which is the case if the full chain is successful, namely if we are able to send back data to earth. If this is the case, the next step is to send a larger detector into space with more layers (up to 30) with two objectives : a mapping of cosmic rays emitted by the sun can be performed using a network of satellites, and a precise mapping of the radiation between the earth and Mars can be performed before sending astronauts to Mars. Obviously, this is high priority for NASA.

\section{MEASURING RADIATION IN CANCER TREATMENT}

The second application that we are going to discuss is related to the measurement of dose delivered to patients when treated for cancer especially for flash beam treatments (using high intensity proton beams as an example) [5]. The same technique applies for beam monitoring in hospitals or also for any commercial or research beam infrastructure.

Our silicon detectors and readout system were put in an electron beam used in the past for radiotherapy at St Luke Hospital, Dublin, Ireland. A schematic of this test is shown in Fig. 7 where we can compare the information from our ultrafast Silicon detector (UFSD) with the usual ion chambers commonly used in hospitals to measure doses delivered to patients. As we will see, our system can provide a precise and instantaneous measurement of dose without the need of calibration and with high spatial resolution (typically $\mathrm{mm}$ ). The new idea is that we have a fast detector that is able to discriminate and count single particles (electrons or protons) benefiting from the fact that the UFSD detector is fast enough.

The first step was to compare the information from our UFSD with traditional measurement of doses using ion

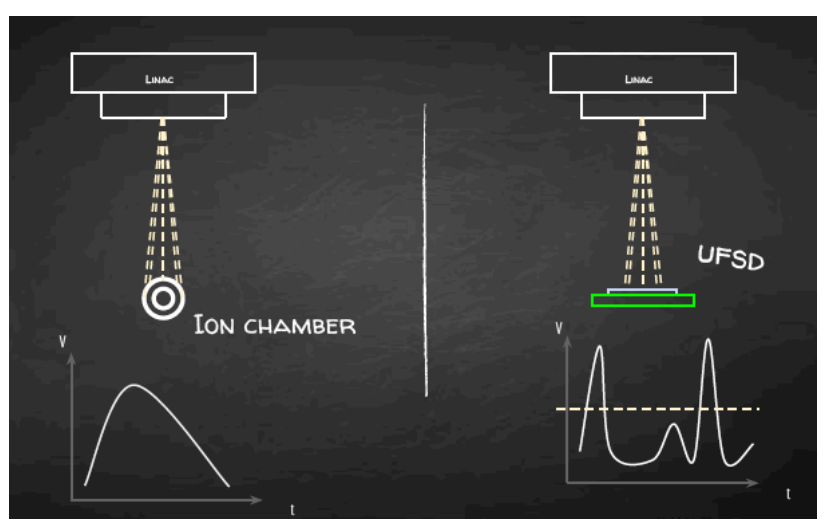

Fig. 7. Schematic of the tests performed at the St Luke hospital at the University of Dublin, Ireland, using an ion chamber or a fast Silicon detector. The signal from the ion chamber is integrated over a few seconds in time whereas the UFSD information can be instantaneous.

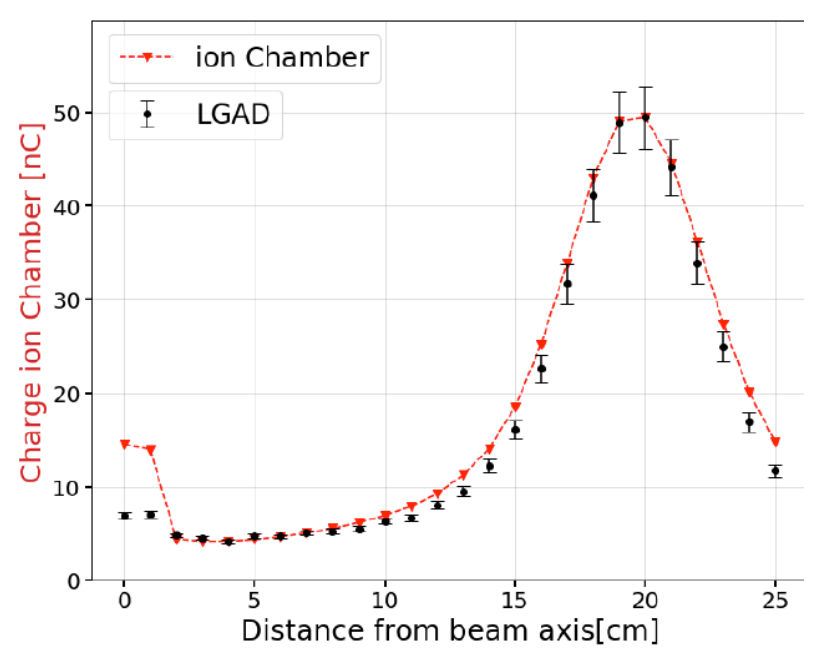

Fig. 8. Comparison between the measurement of the charged ions by the ion chamber (red points) and the measurement using our fast Silicon detector. We note a good correlation between both measurements.

chambers as performed in hospitals. Results are shown in Fig. 8 as a function of distance from the beam axis, downstream a permanent magnet (to create a spectrometer). Results are in very good agreements except at low deviations, that can be explained by the fact that the electron beam is highly contaminated by photons (not affected by the magnetic field) in that region.

The second step was to measure the instantaneous dose delivered to the patient by counting the number of electrons going through our detector. The principle is described in Fig. 9. Each spike in the UFSD is considered as a particle (here an electron) crossing our detector. In the bottom of Fig. 9, we clearly see the signal above the noise that trigger our detector (red triangles) as a function of time. We notice that having a short signal (a few nanoseconds), as well as a very fast sampling method, is crucial to be able to measure each particle crossing the detector. Counting the number of triggered spikes

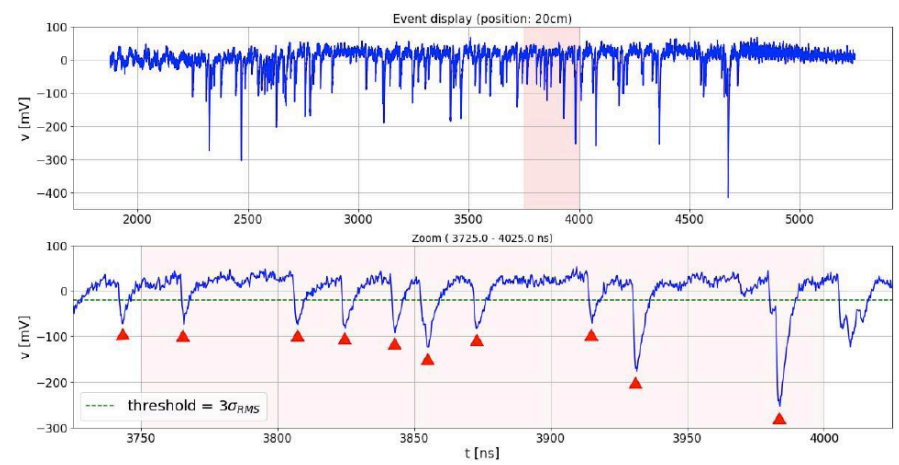

Fig. 9. Signal measured as a function of time. Each spike shows the beam delivered to a potential patient. The red area is zoomed in the bottom plot and the red triangles show the spikes that can be triggered using the Silicon detector. 


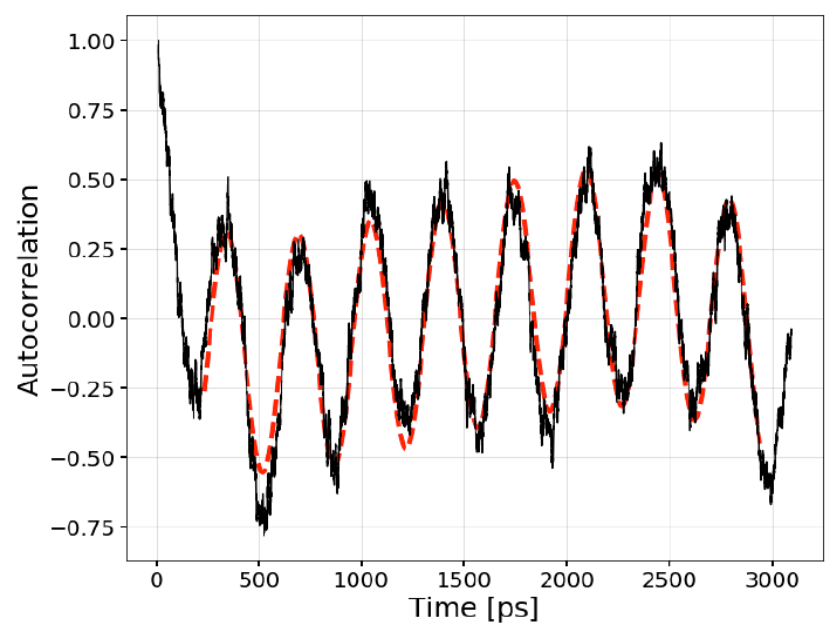

Fig. 10. Bunch structure as measured by the fast Silicon detector. For the first time, we were able to measure the structure in timescales of about 50 ps that allow to measure and control instantaneous doses delivered to patients.

is directly proportional to the dose (with a small efficiency when two consecutive spikes overlap). Fig. 9 clearly shows that this inefficiency is very small and our detector effectively counts all delivered electrons or protons, without the need of temperature and aging calibration, that allow to measure precisely and instantaneously the doses that are delivered to patients during cancer treatment.

Results coming from our UFSD are shown in Fig. 10. We see for the first time the bunch structure delivered by the machine at the St Luke hospital, Dublin. The periodicity of the beam is about 330 ps and measurements using UFSDs can be performed every $50 \mathrm{ps}$. In comparison, the usual ion chamber integrates measurements over times of the order of few seconds, making impossible to use for flash proton therapy. Everyday monitoring of proton beams used for flash proton therapy is also possible using our system.

\section{CONCLUSION}

In this short article, we described two applications using fast Silicon detectors that are used nowadays in a standard way at the LHC.

The first application developed in collaboration with NASA aims at measuring cosmic rays originating from the sun (or other galactic objects) with high precision. Our technique of measuring both the rise time and the amplitude of the signal in the Silicon layer where the particle stops allows identifying the type of particle and measuring its energy using a compact detector that can be sent into space using a cube satellite.

The second application is to measure instantaneously the dose delivered to patients during cancer treatment (especially during flash proton beam therapy). Our technique allows counting the number of particles that are delivered to patients that it is directly proportional to the dose. Another directly related application is everyday monitoring of the beam quality before treatment

Many other applications are also possible using the same technique. It is for instance possible to measure precisely th process of catalysis in chemistry by measuring and analyzing for instance the interface between two liquids or a gas and a liquid and its time variation with a 50 ps precision using interferometry technics. Other applications include beam monitoring for private or public accelerators as well as any instantaneous dose measurements that might be required.

\section{ACKNOWLEDGMENT}

These works come from a collaboration with NASA and the University of Dublin, Ireland, namely F. Gautier, A. Greeley, S.G. Kanekal, T. Isidori, G. Legras, P. McCavana, B. McClean, R. McNulty, N. Minafra, and I would like to thank them for this nice collaboration.

\section{REFERENCES}

[1] A. Apresyan et al., "Studies of uniformity of 50 mum low-gain avalanche detectors at the Fermilab test beam", Nucl. Instr. Meth. A 895 (2018) 158

[2] N. Minafra et al., "Test of ultra-fast silicon detectors for picosecond time measurements with a new multipurpose read-out board", Nucl. Instr. Meth. A 867 (2017) 88.

[3] D. Breton et al., "Measurements of timing resolution of ultra-fast Silicon detectors with the SAMPIC waveform digitizer", Nucl. Instr. Meth. A 835 (2016) 51.

[4] F. Gautier, A. Greeley, S. G. Kanekal, T. Isidori, G. Legras, N. Minafra, A.Novikov, C. Royon, Q. Schiller, “A novel technique for real-time ion identification and energy measurement for in situ space instrumentation", ArXiv:2103.00613,

[5] T. Isidori, P. McCavana, B. McClean, R. McNulty, N. Minafra, C. Royon, "Performance of a low gain avalanche detector in a medical characterization of the beam profile", Phys. Med. Biol. 66135002. 